\title{
Sleep Environment Recommendations for Future Spaceflight Vehicles
}

\section{Zachary A. Caddick'1, Kevin Gregory' ${ }^{1}$, and Erin E. Flynn-Evans ${ }^{2}$ \\ ${ }^{1}$ San Jose State University Research Foundation, NASA Ames Research Center ${ }^{2}$ Human Systems Integration Division, NASA Ames Research Center, Moffett Field CA}

\section{Introduction}

Sleep quality -- including the ability to fall asleep and remain asleep -- and sleep duration are dependent upon circadian phase, length of prior wake duration, and time within the sleep episode ${ }^{[1]}$. Proper alignment of scheduled sleep episodes to the circadian pacemaker is important for sleep consolidation and sleep structure ${ }^{[1]}$.

In order to quantify the impact of a sub-optimal sleep environment on sleep quality and duration, it is important to measure sleep outcomes when sleep is appropriately timed relative to the circadian and homeostatic drives for sleep. It is possible for an individual to experience sleep disruption in an optimal sleep environment due to the imposed sleep schedule. Similarly, it is possible for an individual to experience high sleep efficiency in a sub-optimal sleep environment when accumulated sleep debt is present, which dampens the arousal threshold. Our aim was to compile the evidence associated with sleep disruption due to controllable, environmental stimuli in order to aid NASA engineers and operational personnel in the optimal design of crew sleep accommodations for deep spaceflight.

\section{Methods}

We conducted a comprehensive literature review summarizing optimal sleep hygiene parameters for lighting, temperature, airflow, humidity, comfort, intermittent and erratic sounds, privacy and security in the sleep environment. We reviewed the design and use of sleep environments in a wide range of cohorts including among aquanauts, expeditioners, pilots, military personnel and ship operators. We also reviewed the specifications and sleep quality data arising from every NASA spaceflight mission, beginning with Gemini.

\section{Results}

Sleep Chamber Location. Sleep chambers are key for reducing light and noise pollution. Common areas (e.g. exercise machinery, galley) should be far enough from sleep chambers that sleep periods are not interrupted due to varying crew sleep schedules. Waste management systems in particular can cause sleep disruptions and should be far enough to limit noise exposure, but close enough as to not be an inconvenience to use.
Privacy. Private sleep chambers for each crew member is imperative for a mission's duration. Access to a private space is viewed as critical to the psychological well-being of individuals living in isolated and confined environments. The sleep chambers for deep space vehicles should also allow crewmembers to customize the space with personal items and reconfiguration of stowage compartments.

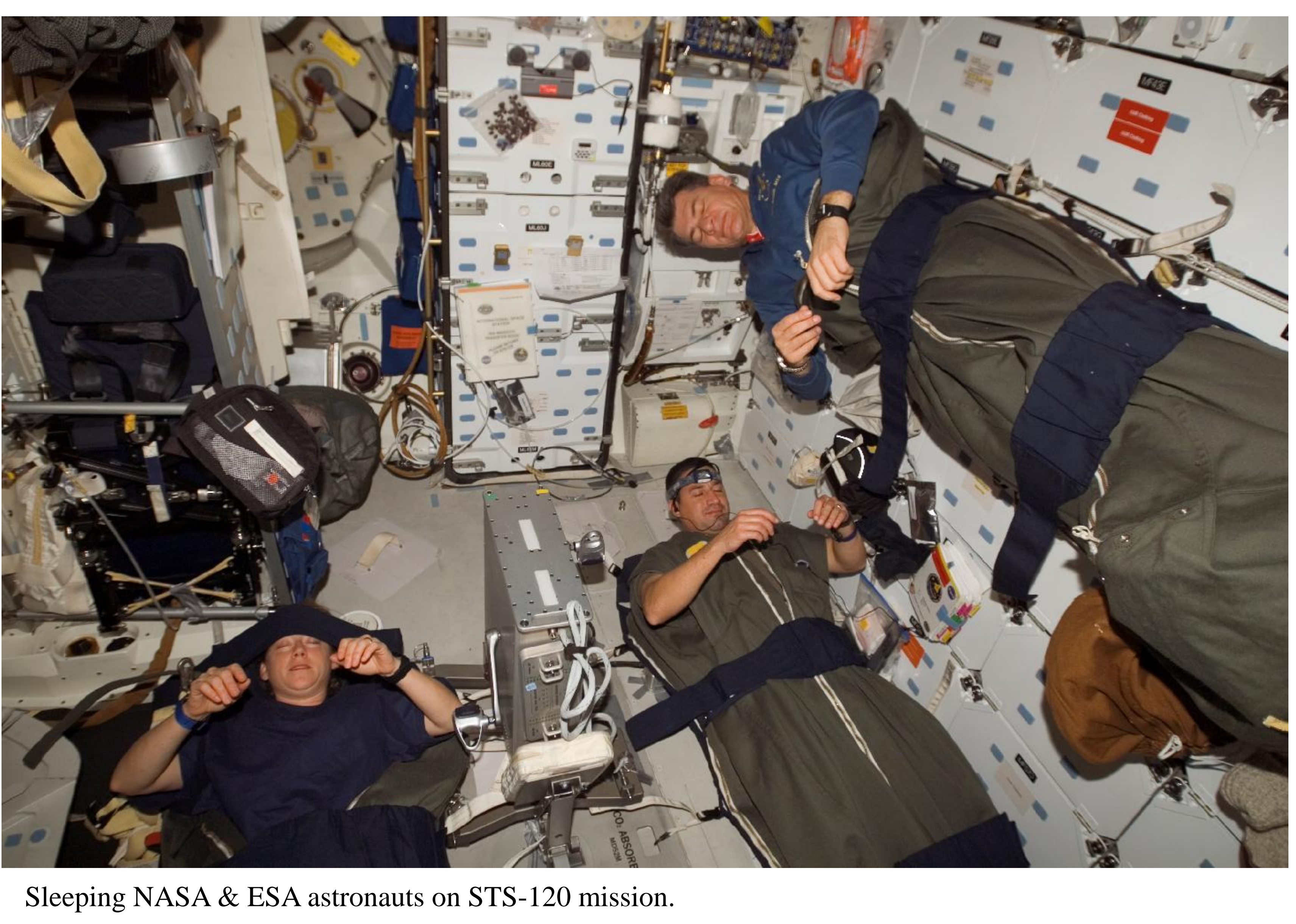

Habitable Volume. The crew quarters that are presently on ISS appear to provide enough habitable volume for crewmembers to move as desired during sleep. We found that individuals living in isolated and confined environments on Earth use their sleep rooms as a place to be alone and to work in addition to sleep ${ }^{[1]}$. As a result, the crew rooms on a planetary excursion should include space for a bed, a desk, and storage of personal belongings.

Light. Light is the primary resetting cue for the human circadian pacemaker ${ }^{[1]}$. Exposure to light at inappropriate times leads to circadian misalignment, which causes sleep disruption [1]. Similarly, exposure to light is alerting and suppresses the drive to sleep [1]. All wavelengths of light have a negative impact on sleep, but blue light elicits the strongest effect due to the stimulation of intrinsically photosensitive retinal ganglion cells [1]. If indicator lights are necessary for identifying egress points, then they should be dim and red ${ }^{[1]}$
Noise. We found that noise has been a major cause of sleep disruption throughout the history of spaceflight. The current NASA guidelines do not provide mitigations against impulsive or intermittent noise ${ }^{[1]}$. We found that exposure to intermittent noise is at least as disruptive to sleep as continuous noise exposure [1]. Exposure to noise be limited to below $35 \mathrm{~dB}$, because exposure to noise above this level is associated with a reduction in sleep quality and quantity, even when individuals do not wake fully ${ }^{[1]}$

Temperature \& Humidity. For optimal sleep, an individual needs to reach his or her thermoneutral equilibrium and should have sufficient bedding available to create a microclimate of between $25-35^{\circ} \mathrm{C}\left(77-95^{\circ} \mathrm{F}\right)^{[1]}$. Given that there are wide individual differences in the optimal temperature for sleep, the sleep environment on future space vehicles should be cool, but there should be sufficient insulation available for crewmembers to modify their environment to suit individual preferences [1] The level of humidity in the environment can also influence sleep quality and quantity. The optimal humidity range for human health is between 40 and $60 \%$ [1].

Air Quality. The optimal ambient gas mixture for sleep is equivalent to the air experienced at sea level on Earth $(78 \%$ nitrogen, $21 \%$ oxygen, $1 \%$ other gases) ${ }^{[1]}$. Similarly, the optimal air pressure during sleep is equivalent to the pressure on the Earth at sea level ${ }^{[1]}$. Air mixtures that deviate from these conditions, such as what mountaineers experience during expeditions, results in disrupted sleep and periodic breathing ${ }^{[1]}$.
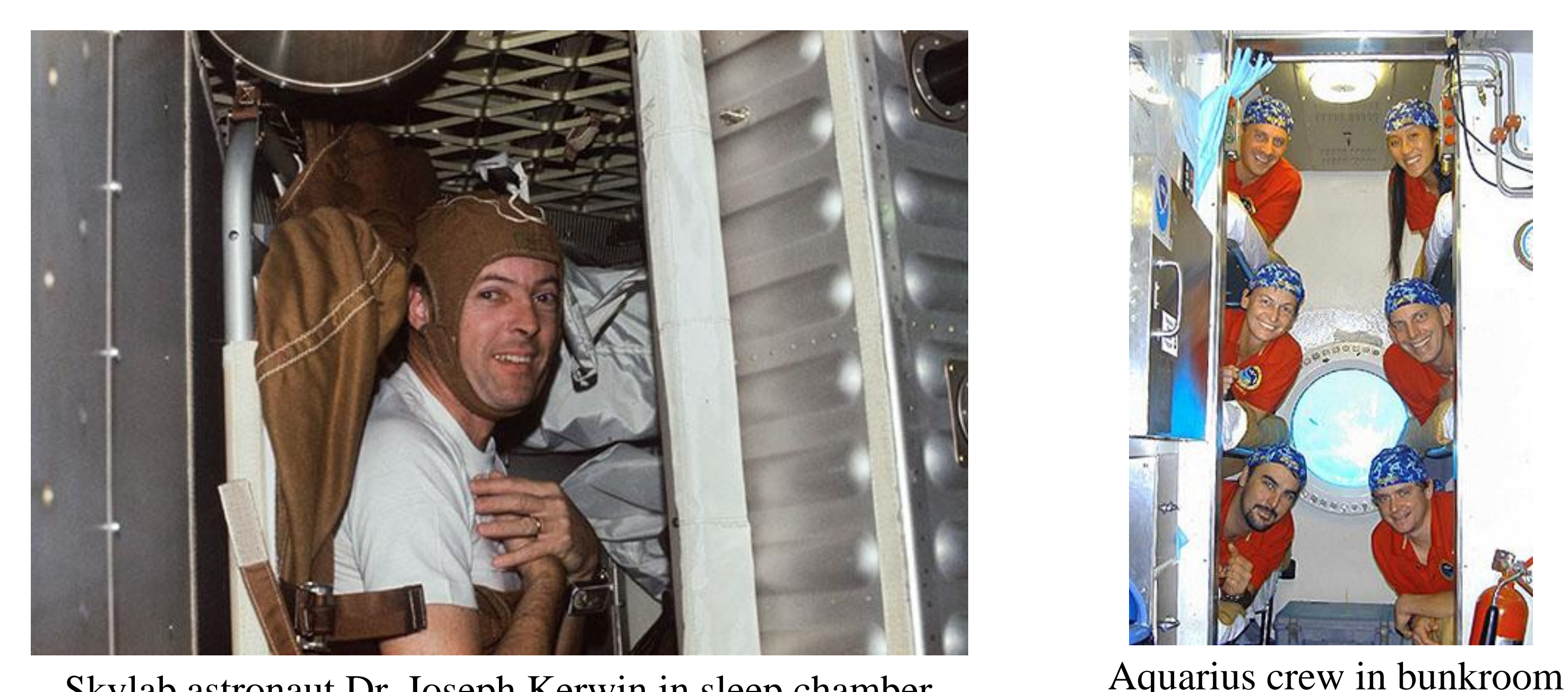

\section{References}

1. Caddick, Z. A., et al. (2016). Sleep environment recommendations for future spaceflight vehicles. 7th International Conference on Applied Human Factors and Ergonomics: Advances in Intelligent Systems and Computing, 484, pp. 923-933. 